\title{
Uplink performance enhancement for LTE licensed-assisted access to unlicensed spectrum
}

\author{
Cheng-Jie Tsai and Tsern-Huei Lee ${ }^{*}$ (D)
}

\begin{abstract}
Licensed-assisted access (LAA) is a new operation mode of carrier aggregation for the Long Term Evolution Advanced systems to transmit data on both licensed and unlicensed spectrum. To deal with the issue of co-existence with WiFi systems, LAA is required to perform listen-before-talk, which can significantly impact its resource utilization. In this paper, the concept called multi-subframe scheduling (MSS) which can enhance the performance of uplink LAA data transmission is presented. MSS allows a user equipment to have multiple channel sensing opportunities and/or to transmit data continuously for multiple subframes. Both scheduling-based and random access schemes are evaluated and compared. The optimum configuration of MSS which maximizes resource utilization of LAA is also provided. Numerical results show that MSS improves resource utilization on the unlicensed spectrum significantly.
\end{abstract}

Keywords: Licensed-assisted access, Resource utilization, Listen-before-talk, Multi-subframe scheduling, Random access

\section{Introduction}

Due to the rapid growth of mobile devices, the amount of mobile traffic increases tremendously. Cisco [1] forecasted that the global mobile traffic will reach 30.6 exabytes (EB; $1 \mathrm{~EB}=10^{18}$ bytes) per month by 2020 . To fulfill such demand, the $5 \mathrm{G}$ cellular systems request 1000 times increase in data rate [2]. The 3rd Generation Partnership Project (3GPP) adopts technologies such as carrier aggregation (CA), multiple antenna, and relaying to boost the data rate for the Long Term Evolution Advanced (LTE-A) systems. One possible approach to further boost the data rate is to deploy small cells which share the same spectrum with macro-cells (i.e., heterogeneous networks). Although data rate increases as the number of small cells increases, a dense deployment of small cells incurs sophisticated inter-cell interference management [3, 4]. As a result, cellular operators consider to exploit unlicensed spectrum together with licensed spectrum to increase the available bandwidth for boosting data rate. One potential direction is the cellular/WiFi interworking $[5,6]$, which allows user equipments (UEs) to adaptively use either the cellular

\footnotetext{
* Correspondence: tsernhueilee@gmail.com

Department of Electrical and Computer Engineering, National Chiao Tung University, Hsinchu, Taiwan
}

systems or the WiFi systems (e.g., IEEE $802.11 \mathrm{a} / \mathrm{n} / \mathrm{ac}$ ). Nevertheless, the interworking requires collaborative management between the two non-interoperable systems. Accordingly, a unified technology framework, named licensed-assisted access (LAA), was proposed for the cellular systems $[7,8]$.

LAA is a new operation mode for the LTE-A systems to transmit data on the unlicensed spectrum. It was first standardized in 3GPP Release 13 for downlink (DL)-only operation [9] and has proceeded to support uplink (UL) operation in Release 14 [10]. Although extending the systems to unlicensed spectrum can benefit from wider bandwidth, several challenges are posted as well. First, communications on unlicensed spectrum could be unreliable, which is particularly harmful to control signal exchanges. Because of the uncontrolled interference, unlicensed spectrum can never match the quality of licensed spectrum regime. To solve this problem, LAA exploits unlicensed spectrum as a complementary tool to augment licensed spectrum based on the framework of CA [11-13]. The primary carrier is the licensed band and is used to carry control signals and QoS-critical data, while the secondary carrier is the unlicensed band and is used to transfer best effort data opportunistically. Consequently, LAA is capable of utilizing unlicensed 
spectrum to boost data rate while providing QoS support, seamless mobility, and good coverage at the same time. Second, communications on unlicensed spectrum must comply with regulations imposed by different countries, such as listen-before-talk (LBT) mechanism where data transmission is forbidden if no prior channel sensing is performed, and limits on maximum channel usage time and maximum transmit power [14]. For example, LBT is mandatory in Japan and Europe for better coexisting with the incumbent systems such as WiFi and radar systems. (We focus on coexisting with WiFi systems in this paper.). To serve as a single global solution which complies with any regional regulatory requirements, LAA specifies LBT as a vital feature to access unlicensed spectrum. The LBT procedure for DL transmission has been specified and the performance was also evaluated in Release 13 [14]. Qualcomm [15] showed that, the total throughput of an LAA operator and a WiFi operator is greater than that of two WiFi operators. In other words, the LAA systems can coexist well with the WiFi systems through the LBT feature.

In Release 14, LAA is tasked to support UL operation. Several facts must be taken into consideration when it comes to support LAA UL. First, the evolved Node B (eNB) has full control of resource allocation and will issue an UL grant before UE can transmit data. Second, UE needs enough processing time to decode an UL grant and prepare data for transmission. The processing time required between an UL grant and the corresponding UL transmission is 4 subframes (i.e., $4 \mathrm{~ms}$ ) in the current standard [16]. The above two principles are designed for eNB to schedule data transmission on licensed spectrum and should also be followed when accessing unlicensed spectrum. Third, to friendly coexist with WiFi systems, UE needs to perform LBT before it can transmit data. If UE detects an idle channel, it can transmit data in the granted subframe; otherwise, it cannot transmit data and has to wait for a new grant. In other words, the actual UL transmission depends on whether there is an idle channel or not at the moment UE is granted to transmit data. As a consequence, the UL transmission may not be conducted as scheduled, which implies the resource utilization of unlicensed spectrum is reduced.

To improve the utilization of LAA UL data transmission, a scheme called multi-subframe scheduling (MSS) was proposed in 3GPP RAN 1 meeting $[17,18]$. Different from the traditional scheduling scheme where a UL grant always indicates the resource allocation on a single subframe, MSS allows a UL grant to indicate resource allocation across multiple subframes. There are two kinds of MSS. The first one is that a UL grant can indicate data transmission on multiple subframes. That is, the UE which receives the grant can transmit data for multiple subframes rather than a single subframe as long as it has found an idle channel before transmission. One may expect an increase of throughput as the number of subframes permitted for transmission increases. The second kind of MSS is that a UL grant can indicate multiple channel-sensing opportunities where UE can transmit data on a single subframe as long as it has found an idle channel in one of the granted opportunities. By allowing more channel sensing opportunities, the probability for a UE to detect an idle channel increases.

For a UE scheduled for data transmission (say, UE 1), the transmission attempt could be a failure if the channel is sensed to be occupied by some WiFi user. However, the interference from this WiFi user to another UE (say, UE 2) could be lower than a given threshold if they are geographically separated apart from each other. In such case, the WiFi transmission will not be interfered by the transmission of UE 2 . As a result, the resource utilization may be improved if a random access scheme is adopted where UL resource is shared by multiple UEs. For example, when UL resource is shared by two UEs, the resource is used for data transmission successfully as long as there is exactly one UE sensing the channel idle and transmitting data after that. Assume that the probability for the channel to be sensed busy for each UE is the same and is denoted by $y$. The utilization of the resource can be calculated as $2 y(1-y)$. When $y>0.5$, the utilization of the resource shared by two UEs can be greater than that used by just a single UE.

In this paper, we evaluate the performance of MSS under scheduling-based and random access schemes. Moreover, the configurations which maximize resource utilization for both scheduling-based and random access schemes are also provided. Numerical results show that MSS improves the resource utilization of unlicensed spectrum significantly for both scheduling-based and random access schemes. Besides, random access outperforms scheduling when WiFi traffic load is heavy.

The rest of this paper is organized as follows. Section II reviews the design principles for LAA UL operation. Section III presents the idea of MSS. In Section IV, we describe the system model. Sections V and VI contain, respectively, performance analysis and optimum configuration of scheduling-based and random access schemes. Numerical results are provided and discussed in Section VII. Finally, we draw a conclusion in Section VIII.

\section{Design principles for LTE uplink access to unlicensed spectrum}

Different to LAA DL transmission, where eNB determines the available channels to avoid transmission collision with WiFi users. In LAA UL transmission, the scheduled resource determined by eNB may conflict with the actual available resource observed at the UE side. Such situation 
leads to inefficient resource utilization and more time needed to complete data transmission.

To improve the resource utilization of LAA UL transmission, we should first understand the design principles of UL operation of the LTE-A systems. The principles designed for data transmission on licensed spectrum should be also followed when accessing unlicensed spectrum. First, eNBs will issue a UL grant before UE can transmit. The UL grant could be carried by either the primary carrier (licensed band) or the secondary carrier (unlicensed band) via the cross-carrier scheduling or the self-scheduling, respectively [11-13]. Note that when the self-scheduling is adopted, eNB can only send a UL grant whenever an idle channel is detected. Second, UE needs enough processing time to decode a UL grant and prepare data for transmission. Based on the requirement of the LTE-A systems, a fixed time gap between a UL grant and the corresponding UL transmission is specified to be $4 \mathrm{~ms}$ in the current standard. That is, a UL grant always indicates resource allocation four subframes after the grant is received [16]. Third, to friendly coexist with WiFi systems, LBT is required to be performed before any transmission. Since there is a time gap between a UL grant and the corresponding UL transmission, UE may not be able to transmit in the granted subframe if LBT fails, i.e., the channel is occupied by WiFi users. Currently, there is no restriction on when to start to perform the LBT procedure [19]. In this paper, we assume that LBT, or clear channel assessment (CCA), is performed right before the beginning of a subframe allocated for data transmission. The time period of performing CCA is $25 \mu \mathrm{s}$, which is much shorter than the length of a subframe (i.e., $1 \mathrm{~ms}$ ) $[8,19]$. During a CCA period, no data transmission is allowed. For each CCA, if UE detects an idle channel, it transmits data in the succeeding granted subframe. Otherwise, UE cannot transmit data in the granted subframe and has to wait for a new grant.

\section{Multi-subframe scheduling}

Due to the mandatory feature of LBT, the resource allocated for UL transmission may not be used if channel is occupied by WiFi systems at the moment UE is about to transmit data. Therefore, the timing relationship between UL grant and the corresponding data transmission is no longer maintained for channel access over unlicensed spectrum. It may result in inefficient resource utilization. In 3GPP Release 14, an idea of MSS is proposed to tackle this problem [18].

There are two kinds of MSS schemes. The first one, referred to as Scheme 1, uses a UL grant to indicate data transmission on $L(L>1)$ subframes as long as the channel is captured. An example of $L=3$ is shown in Fig. 1 . Note that, for convenience, we number in this figure the subframe in which a UL grant is received 0 . (The same numbering method is adopted for the rest of the paper.) In this example, UE is granted to transmit data from subframe 4 to subframe 6 and required to perform CCA right before the beginning of subframe 4 . If UE fails to find an idle channel, it can transmit nothing and has to wait for a new grant from eNB. The major advantage of Scheme 1 is to improve the UL throughput on unlicensed spectrum. The throughput increases as the number of subframes allowed to transmit data increases.

The second kind of MSS scheme, referred to as Scheme 2, allows UE to transmit data in one of $K(K>1)$ consecutive subframes. In other words, after receiving a UL grant, UE can perform CCA, up to $K$ times. An example for $K=3$ is shown in Fig. 2. After receiving a UL grant, UE can perform CCA right before subframes 4,5 , and 6 , to attempt to access channel. If UE captures an idle channel, it transmits in the following subframe and ignores the remaining channel access opportunities, if any. If UE cannot find an idle channel in all of the channel access opportunities, it cannot transmit data and has to wait for a new grant. The major advantage of Scheme 2 is that it reduces latency of requesting UL resource after a CCA failure (i.e., the channel is sensed busy). Also, by providing UE multiple channel access opportunities, the resource utilization can be increased if $K$ is properly selected.

Scheme 1 has been approved in Release 14. The new formats of UL grant are to be specified. The value of $L$ ranges from 2 to 4 . Activation and deactivation of the feature of MSS as well as the value of $L$ are set via Radio Resource Control (RRC) signaling. Note that, by using the formats of UL grant in the current LTE-A systems for $L=1$, Scheme 1 is backward compatible [16, 19]. However, the resource granted in Scheme 1 still remains unsed and causes a wastage if UE cannot find an idle channel before data transmission.

It is straightforward to combine the ideas of Scheme 1 and Scheme 2 to provide UE $K$ channel access opportunities and allow it transmit data for $L$ subframes if the channel is detected idle. In this paper, we denote the combined scheme $S(K, L)$. Clearly, Scheme 1 and Scheme 2 are just $S(1, L)$ and $S(K, 1)$, respectively. In $S(K, L)$, if CCA success (i.e., the channel is sensed idle) happens for the first time in the $i^{\text {th }}$ access opportunity, $1 \leq i \leq K$, then UE transmits data from subframe $4+i-1$ to subframe $4+i-1+L-1$ afterwards.

\section{System model}

In this paper, we evaluate and compare the performance of the combined MSS under both scheduling and random channel access schemes. We will describe the system model in this sub-section first. The performance 


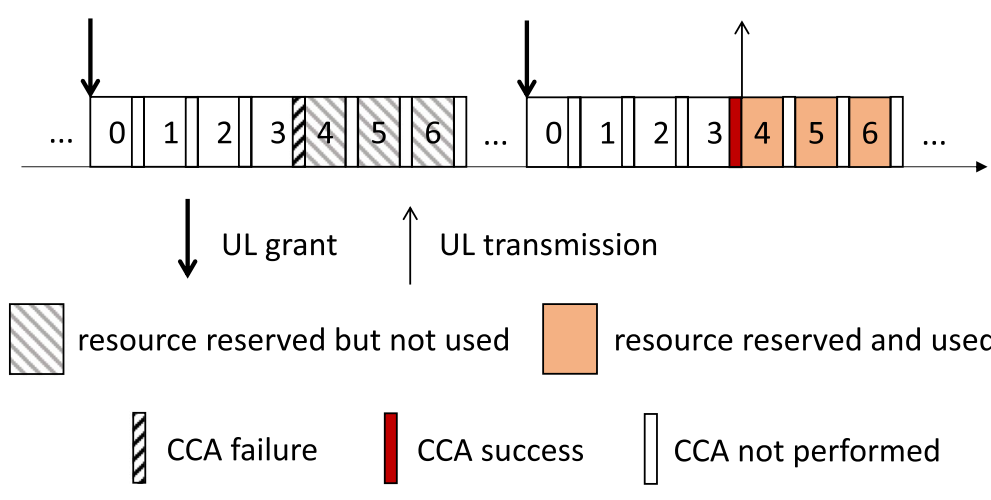

Fig. 1 MSS Scheme 1 forL $=3$

analysis of scheduling and random schemes will be presented in section IV and section V, respectively.

Consider an LAA network which consists of one eNB and $N$ UEs. For simplicity, we consider cross-carrier scheduling where UL grants are sent on the licensed band. The skill of analysis can be applied to selfscheduling. We assume that every UE is always requesting for data transmission and the eNB periodically schedule UEs for UL transmission. Let the period be $\ell$ subframes. $\ell$ must be larger than or equal to $L+K-1$ for $S(K, L)$ because $L$ and $K-1$ subframes are reserved for data transmission and additional CCA opportunities, respectively. For convenience, we call the total reserved subframes the LAA opportunity (LAAOP). It is not hard to see that $\ell$ is a parameter that determines the bandwidth share of LAA. If $\ell>L+K-1$, there is an idle period of length greater than zero between two LAA OPs, during which UE never transmits data. We define a scheduling cycle as an LAA OP followed by an idle period, which can be of length zero if $\ell=L+K-1$. Figure 3 shows an example of $S(2,3)$ when $N=1$ and $\ell$ $=6$. During the LAA OP in a scheduling cycle, UE has $K$ CCA opportunities located in the first $K$ subframes. If the UE finds the channel idle for the first time in the $i^{\text {th }}$ CCA period, it transmits data from subframe $4+i-$ $1(\bmod \ell)$ to subframe $4+i-1+N-1(\bmod \ell), 1 \leq i \leq K$. If the UE does not find the channel idle for all the $K$ CCA opportunities, it cannot transmit data in this scheduling cycle.

For scheduling-based channel access scheme, the eNB assigns a particular UE for potential transmission in each scheduling cycle. The UE assigned for data transimssion performs CCA before the LAA OP begins. If the channel is sensed idle, it transmit data for $L$ ms. Otherwise, it defers data transmission for $1 \mathrm{~ms}$ and performs CCA again after the deferral. It transmits data for $L \mathrm{~ms}$ if the channel is sensed idle and defers for another $1 \mathrm{~ms}$ otherwise. The process repeats until all the K CCA opportunities are used up.

For random access scheme, the eNB assigns all $N$ UEs to contend for potential transmission. All UEs performs CCA right before the biginning of the LAA OP simultaneously. Since a collision will occur if more than one UE find the channel idle and transmit data, we have the following design to reduce the collision probability. For each UE detecting the channel idle, with probability $q$, it decides to transmits data; with probability $1-q$, it decides not to transmit data. If a UE detects the channel to be busy, it defers data transmission for $1 \mathrm{~ms}$ and performs CCA again after the deferral. The process

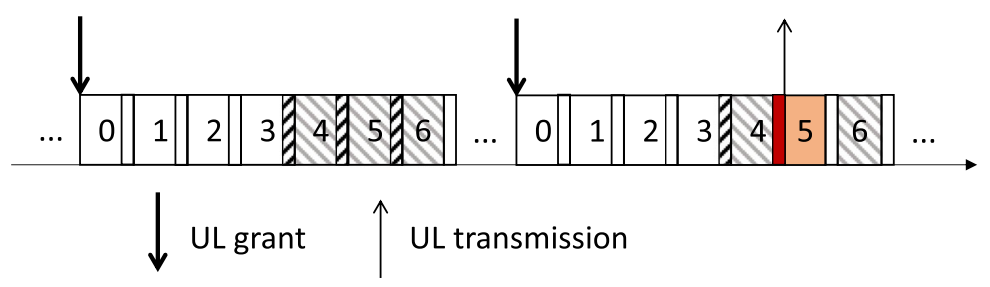

resource reserved but not used resource reserved and used CCA failure $\quad$ CCA success $\rrbracket$ CCA not performed

Fig. 2 MSS Scheme 2 fork $=3$ 


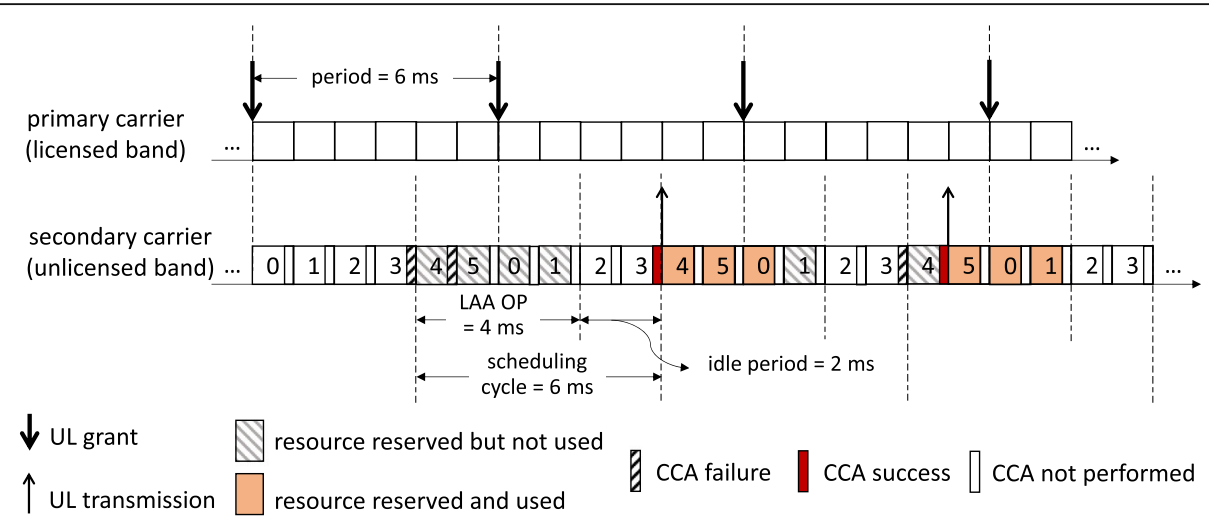

Fig. 3 An example of $S(3,2)$ for $N=1$ and $l=6$

repeats until all the $K$ CCA opportunities are used up. We assume that $1<K \leq L$ so that if a UE starts to transmit its data after the $i^{t h}$ CCA, then the channel will always be sensed busy by other UEs for the rest of CCAs, i.e., CCA $i+1$, CCA $i+2, \ldots$, CCA $K$.

We evaluate the system performance in terms of resource utilization of the LAA network. The utilization, denoted by $\rho$, is defined as the ratio of the average number of subframes successfully used for data transmission to the total number of subframes served as an LAA OP. For each CCA, the impact of interference to the LAA network caused by WiFi networks depends on traffic generated in WiFi networks, the locations of LAA UEs and WiFi users, and channel conditions. It was argued in [20] that the randomness of the above three factors may result in an independently and identically distributed probability of CCA outcome. In this paper, we follow this argument. That is, we assume that the probability for a UE to sense the channel busy is $p$, independent of other UEs and time.

\section{Optimum scheulduling scheme}

\subsection{Evaluation of resource utilization}

The probability that UE transmits data in a scheduling cycle is $\left(1-p^{K}\right)$ which implies the average number of subframes in which UE successfully transmits data in a scheduling cycle is $L\left(1-p^{K}\right)$. Therefore, we have

$$
\rho(K, L)=\frac{L\left(1-p^{K}\right)}{L+K-1}
$$

\subsection{The optimum setting}

In this sub-section, we determine the optimum selection of $K$ and $L$ which maximizes $\rho(K, L)$. We have the following lemmas.

Lemma 1. Given $K, \rho(K, L)$ is an increasing function of $L$.
Proof: We shall prove, for a given $K, \rho(K, L)$ is an increasing function of $L$ by showing that $\rho(K, L+1) / \rho(K$, $L) \geq 1$. We have

$$
\begin{aligned}
\frac{\rho(K, L+1)}{\rho(K, L)} & =\frac{(L+1)(L+K-1)}{L(L+K)} \\
& =\frac{N(L+K+d)+(L+K)-(L+1)}{N(L+K)} \\
& =\frac{L(L+K+d)+K-1}{L(L+K)} \geq 1 .
\end{aligned}
$$

This completes the proof of Lemma 1 .

According to Lemma 1, to increase the resource utilization of the LAA network, $L$ should be chosen as large as possible. In some countries, there is a restriction on the maximum channel occupancy time (MCOT) in each scheduling cycle. For example, in Japan, MCOT is set to be $4 \mathrm{~ms}$ [14]. To maximize $\rho(K, L)$ without violating regulations, the value of $L$ should be chosen to be MCOT.

Lemma 2. It holds that, given $L, \rho(K+1, L)>\rho(K, L)$ if and only if $1+p+\ldots+p^{K}<(L+K) p^{K}$.

Proof: Since $\rho(K, L)=L\left(1-p^{K}\right) /(L+K-1)$, we have

$$
\begin{aligned}
\rho(K+1, N) & >\rho(K, N) \Leftrightarrow \frac{L\left(1-p^{K+1}\right)}{L+K}>\frac{L\left(1-p^{K}\right)}{L+K-1} \\
& \Leftrightarrow\left(1-p^{K+1}\right)(L+K-1)>\left(1-p^{K}\right)(L+K) \\
& \Leftrightarrow p^{K+1}-1>(L+K)\left(1-p^{K}-1+p^{K+1}\right) \\
& \Leftrightarrow 1-p^{K+1}<(L+K) p^{K}(1-p) \\
& \Leftrightarrow 1+p+\ldots+p^{K}<(L+K) p^{K} .
\end{aligned}
$$

Therefore, Lemma 2 is true. Note that in the above proof, the symbol $\Leftrightarrow$ denotes if and only if.

Lemma 3. Given $L, \rho(K, L) \leq \rho(K-1, L)$ implies $\rho(K+$ $1, L) \leq \rho(K, L)$. 
Proof: Assume that $\rho(K, L) \leq \rho(K-1, L)$.According to Lemma 2 , it is true that $1+p+\ldots+p^{K-1} \geq(L+K-1) p^{K-1}$. Since $(L+K-1) p^{K-1} \geq(L+K-1) p^{K}$, we have

$$
\begin{aligned}
1+p+\ldots+p^{K} & =\left(1+p+\ldots+p^{K-1}\right)+p^{K} \\
& \geq(L+K-1+d) p^{K}+p^{K} \\
& =(L+K) p^{K},
\end{aligned}
$$

which, according to Lemma 2 again, implies that $\rho(K+1$, $L) \leq \rho(K, L)$. End of proof, Lemma 3.

Based on Lemma 1 to Lemma 3, we have the following theorem for finding the optimum values of $K$ and $L$ which maximize $\rho(K, L)$.

Theorem 1. The optimum values of $K$ and $L$, denoted by $K^{*}$ and $L^{*}$, which maximize $\rho(K, L)$ satisfy $L^{*}=$ MCOT and $K^{*}=\min \left\{k \mid \rho\left(k+1, L^{*}\right) \leq \rho\left(k, L^{*}\right)\right\}$.

\section{Optimum random access scheme}

In this section, we first analyze the performance of the simplest scheme $S(1,1)$ and then generalize the analysis to scheme $S(K, L)$.

\subsection{Performance analysis of $S(1,1)$}

Let $x$ be the probability for a UE not to transmit data after performing CCA. It happens when a UE finds the channel busy or decides not to transmit data after detecting the channel idle. Therefore, we have $x=p+(1-$ $p)(1-q)=1-q+p q$. An LAA OP is successfully used when there is exactly one UE which finds the channel idle and decides to transmit data. The probability for this case is $C_{1}^{N}(1-x) x^{N-1}=N(1-x) x^{N-1}$, where $C_{y}^{x}$ $=\frac{x !}{(x-y) ! y !}$. The utilization is then given by

$$
\rho(1,1)=N(1-x) x^{N-1}
$$

Let $q^{\prime \prime}$ be the value of $q$ which maximizes the utilization. Denote by $\rho^{\prime}(1,1)$ the derivative of $\rho(1,1)$ with respect to $q$. We have

$$
\rho^{\prime}(1,1)=N(p-1) x^{N-2}(N-1-N x) .
$$

Note that $\rho^{\prime}(1,1)=0$ holds when $q=q^{*}$. Given $p \neq 1$, to solve $\rho^{\prime}(1,1)=0$, we have $N-1-N x=0 . q^{\prime \prime}$ can then be solved as follows.

$$
q^{*}=\left\{\begin{array}{c}
1, \text { if } N(1-p)<1 \\
\frac{1}{N(1-p)}, \text { otherwise }
\end{array}\right.
$$

Eqn. (6) can be rewritten as follows.

$$
q^{*}=\min \left(1, \frac{1}{N(1-p)}\right)
$$

Note that $N(1-p)$ is the average number of UEs detecting the channel idle and $q^{*}=1 / N(1-p)$ when $N(1$ $-p) \geq 1$ sets the average number of UEs that transmits data to be 1 .

The maximum utilization achieved by setting $q=q^{*}$ is given in the following theorem.

Theorem 2. The maximum utilization achieved by the random access scheme sharing by $N$ UEs is given by

$$
\rho^{*}=\left\{\begin{array}{c}
N(1-p) p^{N-1}, \text { if } N(1-p)<1 \\
\left(\frac{N-1}{N}\right)^{N-1}, \text { otherwise }
\end{array}\right.
$$

Proof: Eq. (8) is obtained by substituting Eq. (7) into Eq. (5).

When $N(1-p) \geq 1, \rho^{*}$ is only determined by $N$. As $N \rightarrow \infty$, we have $\rho^{*} \rightarrow e^{-1}$, which is the same result obtained for the slotted ALOHA scheme. However, when $p$ is too large so that the average number of UEs detecting the channel idle is less than one (i.e., $N(1-p)$ $<1)$, the utilization can be lower than $e^{-1}$ since it is very likely that there is no UE transmitting data after performing CCA.

Because of multi-user diversity, the resource utilization may be increased if it is shared by multiple UEs. On the contrary, transmission collision in the random access scheme can reduce the utilization. As such, it is natural to ask under what condition the random access scheme performs better than the scheduling-based scheme. We have the following theorem. Recall that the utilization of the scheduling-based scheme for scheme $S(1,1)$ is $1-p$. The proof of Theorem 3 is straightforward and thus is omitted.

Theorem 3. The random access scheme performs better than the scheduling-based scheme if and only if

$$
\begin{aligned}
N p^{N-1} & >1 \text { when } N(1-p)<1 \text { or }\left(\frac{N-1}{N}\right)^{N-1} \\
& >1-p \text { otherwise. }
\end{aligned}
$$

\subsection{Performance analysis of $S(K, L)$}

As defined in part A, $x=1-q+p q$ is the probability for a UE not to transmit data after a CCA. The allocated resource is successfully used when exactly one UE finds the channel idle and decides to transmit data. The probability for this case is $C_{1}^{N}(1-x) x^{N-1}\left[1+x^{N}+x^{2 N}+\ldots\right.$ $\left.+x^{(K-1) N}\right]=N(1-x) x^{N-1} \frac{1-x^{K N}}{1-x^{N}}$. Besides, the total number of subframes served as an LAA OP in $S(K, L)$ is $L+K-1$. Therefore, the utilization is given by 


$$
\rho(K, L)=\frac{L N(1-x) x^{N-1}\left(1-x^{K N}\right)}{(L+K-1)\left(1-x^{N}\right)}
$$

Assume that $L$ is set to be MCOT. We now determine the values of $K$ and $q$ which maximize $\rho(K, L)$ when there are $N$ UEs sharing the UL resource. Let $K^{\prime \prime}$ and $q^{*}$ denote, respectively, the values of $K$ and $q$ which together maximize $\rho(K, L) . K^{\prime \prime}$ and $q^{\prime \prime}$ can be obtained by comparing $\rho(K, L)$ for all possible combinations of $K$ and $q$. If there are at least two combinations which result in the maximum value of $\rho(K, L)$, we randomly select one of these combinations for $K^{\prime \prime}$ and $q^{\prime \prime}$.

\section{Numerical results}

In this section, we evaluate the performances of MSS under scheduling-based and random access schemes. Although the maximum allowable value of $L$ currently agreed in Release 14 is 4 , we provide results for $L$ larger than 4 to better illustrate the impact of $L$ on resource utilization. We consider a system with $N=10$.

\subsection{MSS under scheduling-based scheme}

\subsubsection{The effects of $K$ and $L$}

Figure 4 shows the utilization of scheme $S(K, L)$ when $p$ $=0.4$. As was proved in Lemma $1, \rho(K, L)$ is an increasing function of $L$ given $K$. When $K=1, \rho(1, L)=1-p$ is a constant and is independent of $L$. Moreover, given $L$, there is a value $K^{\prime \prime} \geq 1$ such that $\rho(K-1, L)<\rho(K, L)$ if $K$ $<K^{*}$ and $\rho(K+1, L) \leq \rho(K, L)$ if $K \geq K^{*}$. This property was proved in Lemma 3.

\subsubsection{The effect of $p$}

Figure 5 shows $\rho(K, 10)$ for various values of $p$. One can see that given $K, \rho(K, 10)$ decreases as $p$ increases. Moreover, $K^{n}$ increases as $p$ increases. This is because LAA needs more CCA opportunities to capture the channel in a scheduling cycle under heavy traffic load. Analytical

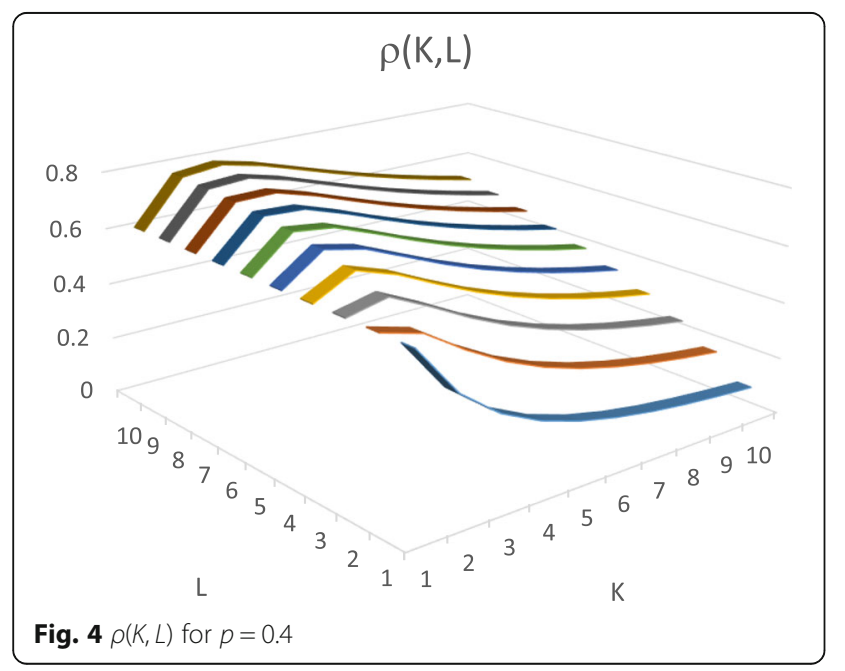

results show that $K^{\prime \prime}=10,3$, and 2 for $p=0.9,0.5$, and 0.2 , respectively. Therefore, we conclude that providing multiple CCA opportunities does improve resource utilization, especially when the traffic load of WiFi systems is high.

\subsection{MSS under random access scheme 7.2.1 The effects of $K$ and $L$}

Figure 6 shows the utilization of $S(K, L)$ under the random access scheme for different values of $K$ and $L$ when $p=0.4$. We assume that $K \leq L$ for the random access scheme. As one can see, given $K>1, \rho(K, L)$ increases as $L$ increases. When $K=1, \rho(1, L)=N(1-x) x^{N-1}$ is a constant, independent of $L$. According to numerical results, there exist $K^{\prime \prime} \geq 1$ such that $\rho(K-1, L)<\rho(K, L)$ if $K<K^{\prime \prime}$ and $\rho(K+1, L) \leq \rho(K, L)$ if $K \geq K^{*}$. Moreover, allowing more channel sensing opportunities does improve utilization. When the value of $K$ is large, excessive subframes are scheduled but not used for transmission, leading to degradation of utilization.

\subsubsection{The effect of $p$ and $q$}

Figure 7 shows $\rho(K, 10)$ for different values of $K$ and $q$. In general, $\rho(K, 10)$ decreases as $q$ increases for a small value of $p$. The reason is that most UEs sense the channel idle for a small $p$ and hence the probability of transmission collision increases as $q$ increases. However, there are exceptions. As an example, for $p=0$ (see Fig. 7a), all 10 UEs sense the channel idle. Consider the case $K=1$. When $q=0.1$, the average number of UEs which transmit data is equal to 1 and the utilization is maximized. As can be seen in Fig. 7a, the utilization for $q=0.05$ is smaller than that for $q=0.1$ for $K=1$. For large values of $K$, the utilization for $q=0.05$ is greater than that for $q=0.1$ (see Fig. 7a for $K \geq 3$ ). Consider the case $K=3$. The probability of successful transmission is equal to $10 q(1-q)^{9}\left[1+(1-q)^{10}+(1-q)^{20}\right]$. It turns out

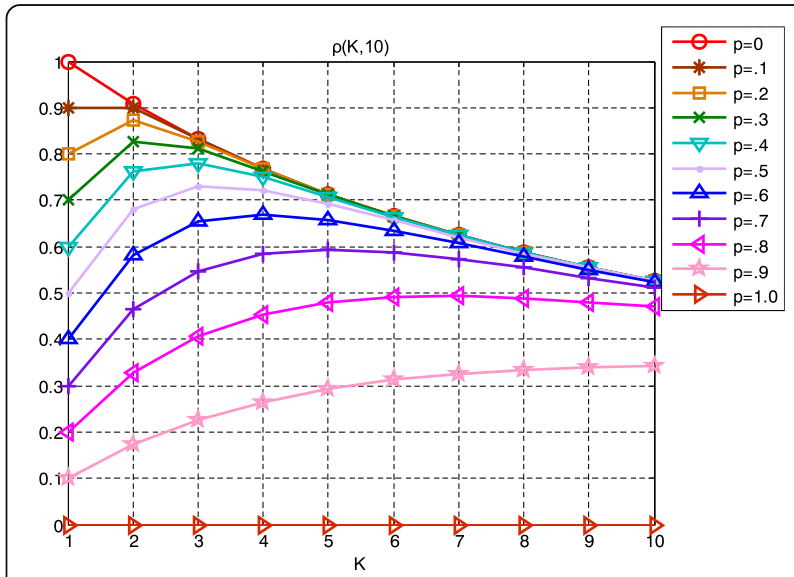

Fig. $5 \rho(K, 10)$ for different values of $p$ 


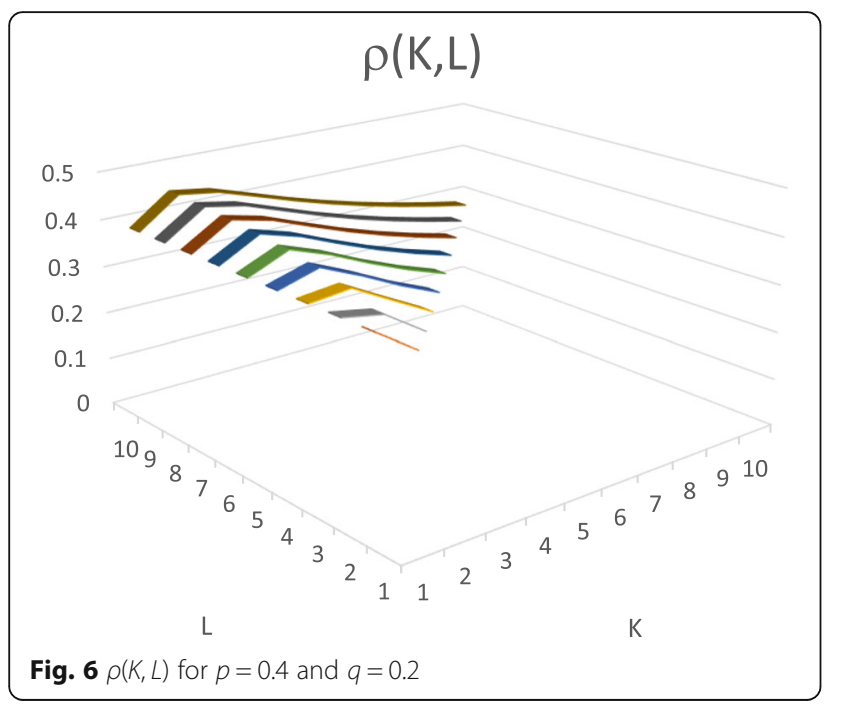

that $q=0.05$ results in a larger value of successful transmission probability than $q=0.1$.

For large values of $p, \rho(K, 10)$ may increase as $q$ increases. The reason is that, when $p$ is large, the number of UEs detecting the channel idle tends to be small. As a consequence, a large value of $q$ can increase the average number of transmission attempts to improve utilization. This phenomenon can be seen for $K=1 \sim 4$ in Fig. $7 d$ where $p=0.8$. In this region, $q=0.1$ yields a better utilization than $q=0.05$.

An observation from Fig. 7 is that $q$ should be small for very large values of $K$. This is not a surprise because any transmission collision will destroy many unused channel access opportunities. To avoid transmission collision, one should use a small value of $q$.

\subsection{Comparison of scheduling-based scheme and random access scheme}

Figure 8 shows the optimum utilizations of MSS under scheduling-based and random access schemes when $L=$ $4 \mathrm{~ms}$. For a given $p$, the maximum utilization is obtained by selecting the best value of $K$ for the scheduling-based scheme or the best combination of $K$ and $q$ for the random access scheme. Scheduling preforms better than random access for small values of $p$. This is due to transmission collision among multiple UEs under random access. For large values of $p$, resource sharing by multiple UEs becomes a better choice than scheduling because of multi-user diversity. As an example, consider the simple system with $K=1$. If only one UE is allowed to access the resource, then the probability that it is not blocked by WiFi users is given by $1-p$. The probability that the resource is used by some UE becomes $n \cdot(1-p) \cdot p^{n-1}$ if $n$ UEs are allowed to access the UL resource. It is clear
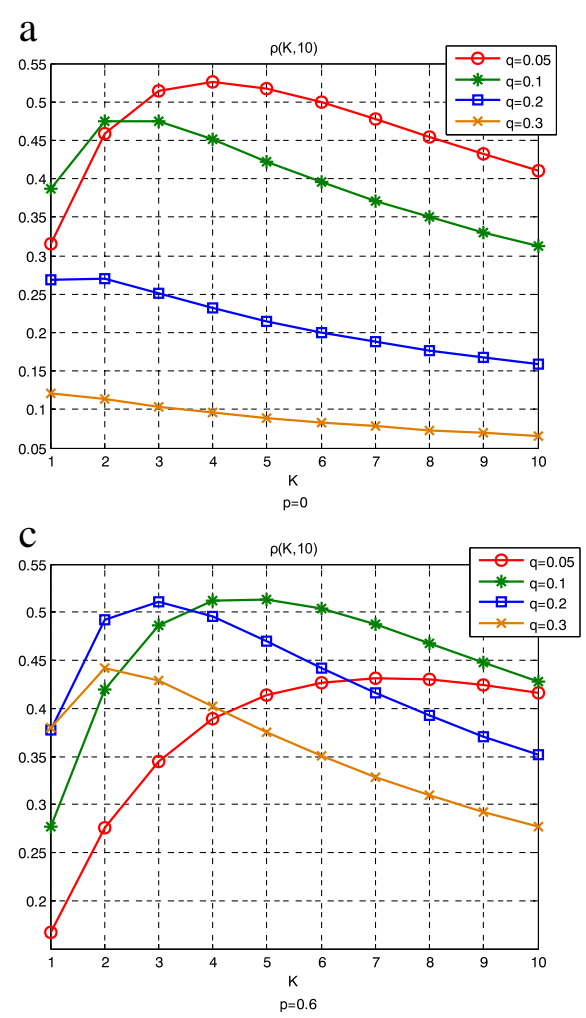

Fig. $7 \rho(K, 10)$ for $(\mathbf{a}) p=0$, (b) $p=0.4,(\mathbf{c}) p=0.6$, and (d) $p=0.8$ b

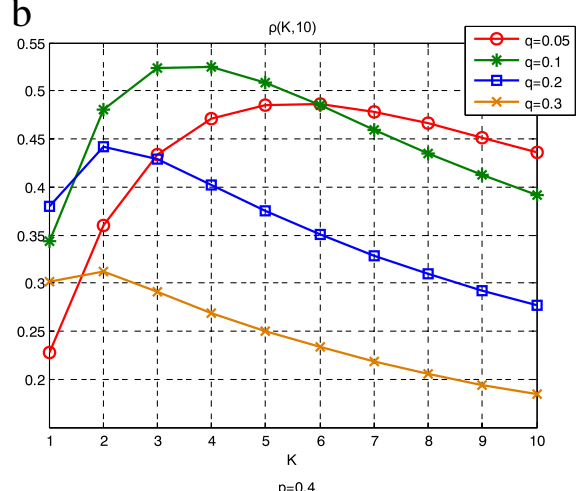

d

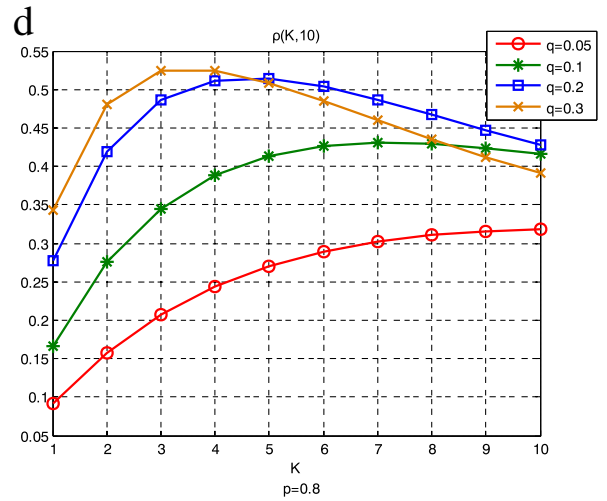




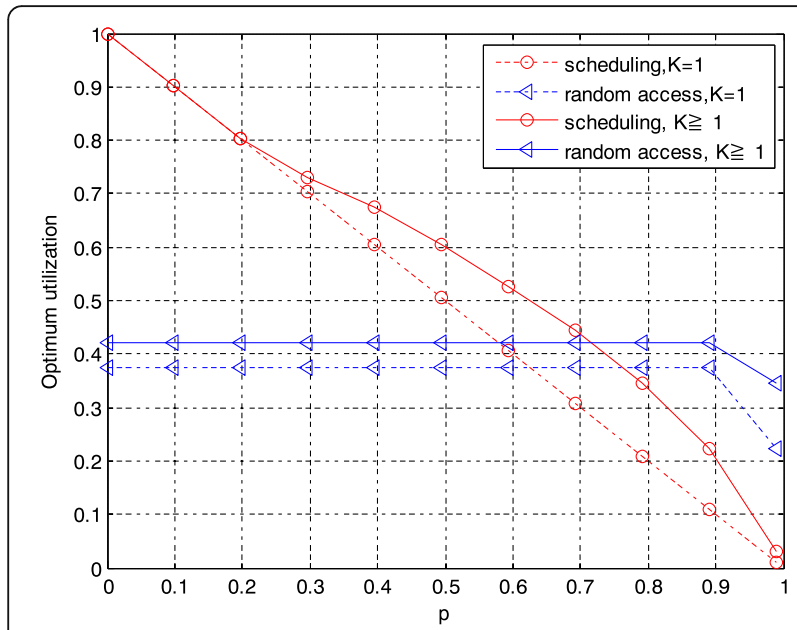

Fig. 8 Optimum utilization for MSS under scheduling-based and random access schemes

that $1-p<n \cdot(1-p) \cdot p^{n-1}$ if and only if $p>(1 / n)^{1 /(n-1)}$. This explains multi-user diversity does improve utilization for large values of $p$..

For comparison, we also show in Fig. 8 the optimum performances for $K=1$. As one can see, allowing multiple CCA opportunities does improve system utilization.

\section{Conclusion}

In LAA, the resource allocated but was not actually used because of WiFi users reduces its utilization. To cope with the problem, the concept of MSS was approved in Release 14 to improve resource utilization. In this paper, a family of MSS schemes is presented and evaluated. Some properties are proved and used to determine the optimum scheme which maximizes utilization. We show that allowing UE to transmit data continuously for multiple subframes always increases utilization. However, providing UE more opportunities to perform CCA does not always increase utilization. This is because more CCA opportunities means longer scheduling cycle which tends to decrease utilization, especially under light WiFi traffic load. Numerical results reveal that, due to multiuser diversity, random access outperforms scheduling under extremely high WiFi traffic load condition.

\section{Acknowledgements}

The research presented in this paper was supported by Ministry of Science and Technology, Taiwan, under grant MOST 104-2221-E-009-086-MY2.

\section{Authors' contributions}

C-JT proposed the design of MSS under both scheduling-based and random access schemes. She also perform numerical analysis and simulations. T-HL suggested the approach of mathematical analysis. Both authors read and approved the final manuscript.

\section{Competing interests}

The authors declare that they have no competing interests.

\section{Publisher's Note}

Springer Nature remains neutral with regard to jurisdictional claims in published maps and institutional affiliations.

Received: 12 October 2017 Accepted: 12 January 2018

Published online: 22 January 2018

\section{References}

1. Cisco whitepaper. Cisco Visual Networking Index: Global Mobile Data Traffic Forecast Update, 2015-2020 (2016).

2. JG Andrews, S Buzzi, W Choi, SV Hanly, A Lozano, ACK Soong, JC Zhang, What will 5G be? IEEE J. Selected Areas Commun. 32(6), 1065-1082 (2014).

3. H Zhang, S Chen, X Li, H Ji, X Du, Interference management for heterogeneous networks with spectral efficiency improvement. IEEE Wirel. Commun. 22(2), 101-107 (2015).

4. V Jungnickel, K Manolakis, W Zirwas, B Panzner, V Braun, M Lossow, M Sternad, R Apelfröjd, T Svensson, The role of small cells, coordinated multipoint, and massive MIMO in 5G. IEEE Commun. Mag. 52(5), 44-51 (2014).

5. M Bennis, M Simsek, A Czylwik, W Saad, S Valentin, M Debbah, When cellular meets WiFi in wireless small cell networks. IEEE Commun. Mag. 51(6), 44-50 (2013).

6. S Andreev, M Gerasimenko, O Galinina, Y Koucheryavy, N Himayat, S-P Yeh, $S$ Talwar, Intelligent access network selection in converged multi-radio heterogeneous networks. IEEE Wirel. Commun. 21(6), 86-96 (2014).

7. R Zhang, M Wang, LX Cai, Z Zheng, X Shen, L-L Xie, LTE-unlicensed: the future of spectrum aggregation for cellular networks. IEEE Wirel. Commun. 22(3), 150-159 (2015).

8. A Mukherjee, J-F Cheng, S Falahati, H Koorapaty, DH Kang, R Karaki, L Falconetti, D Larsson, Licensed-assisted access LTE: coexistence with IEEE 802.11 and the evolution towards 5G. IEEE Commun. Mag. 54(6), 50-57 (2016).

9. 3GPP RP-141664, Study on Licensed-Assisted Access Using LTE, Ericsson, Qualcomm, Huawei, and Alcatel-Lucent, (2014).

10. 3GPP RP-152272, New Work Item on Enhanced LAA for LTE, Ericsson and Huawei, (2015).

11. 3GPP TS 36.300 v14.0.0, Evolved Universal Terrestrial Radio Access (E-UTRA) and Evolved Universal Terrestrial Radio Access Network (E-UTRAN); Overall Description, Stage 2 (Release 14), (2016).

12. 3GPP TR 36.814 v9.0.0, Evolved Universal Terrestrial Radio Access (E-UTRA): Further Advancements for E-UTRA Physical Layer Aspects (Release 9), (2010).

13. 3GPP TR 36.912 v13.0.0, Feasibility Study for Further Advancements for EUTRA (LTE-Advanced) (Release 13), (2015).

14. 3GPP TR 36.889 v13.0.0, Study on Licensed-Assisted Access to Unlicensed Spectrum (Release 13), (2015).

15. Qualcomm whitepaper. Qualcomm Research LTE in Unlicensed Spectrum: Harmonious Coexistence with Wi-Fi (2014).

16. 3GPP TS 36.213 v14.0.0, Evolved Universal Terrestrial Radio Access (E-UTRA); Physical Layer Procedures (Release 14), (2016).

17. 3GPP R1-165235, Uplink Channel Access Mechanism in eLAA, III, (2016).

18. 3GPP R1-161409, Way Forward on Multi-Subframe Scheduling in LAA, LG Electronics, Qualcomm, and ZTE, (2016).

19. 3GPP R1-166056, Final Report of 3GPP TSG RAN WG1 \#85 v1.0.0, (2016).

20. SY Lien, J Lee, YC Liang, Random access or scheduling: optimum LTE licensed-assisted access to unlicensed spectrum. IEEE Commun. Lett. 20(3), 590-593 (2016)

Funding

Not applicable. 